\title{
From Space to Interface: Augmented Reality, a Model for Participation
}

\author{
Liam Noah Jefferies \\ University of Sunderland \\ St Peters Campus, UK \\ Liam.jefferies@me.com
}

\begin{abstract}
Interaction with one's peers and the sharing of experience is a powerful means of generating meaning and creating lasting impression. This paper focuses upon the conceptual and practical development of a participatory artwork that utilises Augmented Reality technology. Using AR as the medium, the aim is to create a generative and interactive artwork that facilitates participation and viewer collaboration. Thereby, one can offer an alternative to the recognised digital interactives that proliferate within both the art and heritage sectors and seeking to use this model as a means to promote interpersonal and social interaction.
\end{abstract}

Augmented reality. Participation. Interaction. Arts. New media. Digital.

\section{1: INTRODUCTION}

Contact, reaction and interaction, with our peers and companions is indelibly linked to what is experienced and how we behave, which is, of course as true, within the gallery or museum, as anywhere else (Falk \& Dierking 1997; Heath \& Lehn 2010). It is however, of particular concern, to curators and museum professionals whose business and in many cases business model, is so involved with issues such as audience engagement and audience growth. 'Interactivity' and increasingly, 'Participatory', have become catchphrases of the museum and gallery, and more often than not it is new media that is called upon to deliver this.

\section{2: RESEARCH}

New Media, or digital interventions within museums and galleries are most commonly associated with info-graphics or curatorial aids (Tsoroni 2009) and have for a long time been the basis for what are nominally called interactives. This is not meant to imply that all interactives are essentially digital, there are some great examples in the science museum (London) that are purely mechanical and very engaging at that. It is however true that for the most part an interactive intervention takes the form of a digital display upon which a single user navigates a set of scenarios that are, in someway linked to the adjacent physical objects.

Through the use of recognised technological interfaces within these displays, be that touch screen or mouse and keyboard, there is an inherent restriction upon the process of engagement and the control of interaction. 'The model places the individual and the individual's interactions with the artefact or system at the heart of the agenda' (Heath \& Lehn 2010) removing the social context of the event and reducing the connections and opportunities for shared experience. Galleries, are filled with interactives that promote a culture of isolation, people on their own, interacting with a system to complete a particular task.

It is not within the scope of this paper to fully explore modes of learning and engagement within the museum or gallery. There is a wide array of writing that covers this, with many opposing points of view and many a division between the ideals and manifesto's of 'traditional and new style exhibitions' and those who both curate and visit them (Griggs 1990). It is however important within this context to consider audience experience and the meaning visitors make of their museum or gallery visit and the outcomes that are achieved. Be this simply measured as greater 'dwell time', by repeat visits, or more intangible as lasting impressions or powerful epiphanies from audiences (Hein 1998).

As intimated above it is becoming increasingly recognised that the social and interpersonal relationships and the collaborative actions of viewers to a gallery or museum plays a vital role in creating lasting impressions and helps to deliver better experiences. It is also becoming apparent that many of the tools, which are technological in 
the majority, with which curators attempt to facilitate and encourage interactivity, do so at the expense of social and collaborative interaction (Heath et al. 2005).

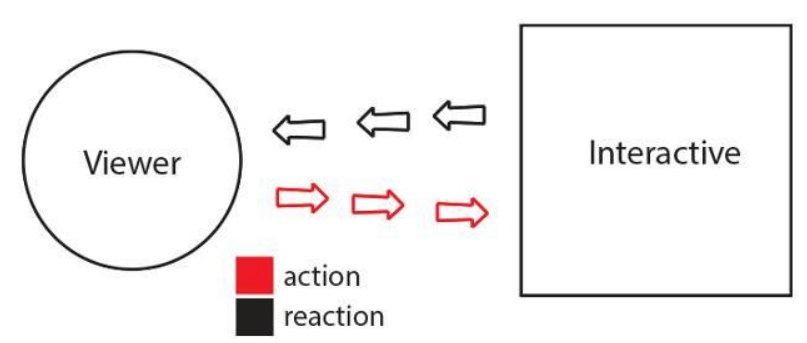

Figure 1: Diagram illustrating a traditional participant's interactions with a system (By the author, 2015)

The process of removing the viewer from their social context in order to interact with a system is not restricted to informatics and curatorial displays. Much of the new media artworks that are overtly positioned as interactive, of which many were showcased at the digital revolutions exhibition at the Barbican in 2014 are developed using the same principals. An example of this would be Chris Milk's 'The Treachery of Sanctuary' (2012) which will be familiar to many due to amount of images viewers posted of their children using it on social media.

This piece however awe-inspiring in terms of scale was designed to interact with a single participant, inevitable queues formed, forcing those wanting to try it to wait. The artwork itself takes each viewer through 3 stages of transformation. In each case the same actions elicit the very same result, growing wings and flying out of shot for example. Perhaps due to the busyness of the show the viewer was immediately instructed by a gallery attendant on the correct physical action that would trigger each of the three phases of the work. This explicit instruction both reduced the time that each person spent upon the interaction and negated any experimentation or play with the artwork and its particular interactive process.

It is however the physical experience of viewing this work that was the biggest barrier to social interaction, those who wished to take part where required to wait in a side gallery, behind a curtain, to prevent them from witnessing the event and ruining the surprise. Those who wished to observe the spectacle of their companions flying away were required to wait behind a barrier at the back of the space, separated from the participant.

Milk states in an interview with the Guardian from June last year that 'the ultimate challenge is how to remove the interfaces that are "an emotional barrier" between the viewer and the work' (Parkin 2014). In the interview this relates to mouse and keyboard, but fails to recognise the impacts of the physical interface and the barriers that are inherent to interaction with this artwork and the effect this has upon the lasting experiences offered.

It was this pervasion of single participant focused interactivity within artworks that were seeking to move beyond traditional interface models that motivated the experimental project that is being demonstrated today. Through the use of a BOYD (bring your own device) model, Augmented reality, image targets and virtual buttons, the number of direct participants is restricted only by space and the quantity of people who are willing to engage. This combined with the generative and non-linear progression of the possible outcomes gives the impression of endless possibility and offers a model for the creation of a participatory experience that necessitates social interaction and group participation.

In this project each viewer can be the director of events, but these events rely upon others. The artwork is designed to require multiple participants in order to fully expose the complexity of the digital object. Those participating are unaware of the reaction of the artwork to their actions, viewers are instrumental and collaboration is essential to explore the potentials of the piece. This is a departure from the majority of new media interactives that proliferate within both the heritage and art institutions with Rafael Lozano-Hemmer's 2008 piece "under scan" being the most notable exception.

Social interaction and collaborative action plays an important role in the connections and experiences delivered during a visit to a cultural event. Many interventions that are seen to develop these connections through the interactive processes they embody are in fact reducing the level interpersonal interaction that would occur if these interventions had not been made. With certain curatorial interventions seen, by some, to detract from rather than augment the experience of the viewer (Huhtamo 2010). 


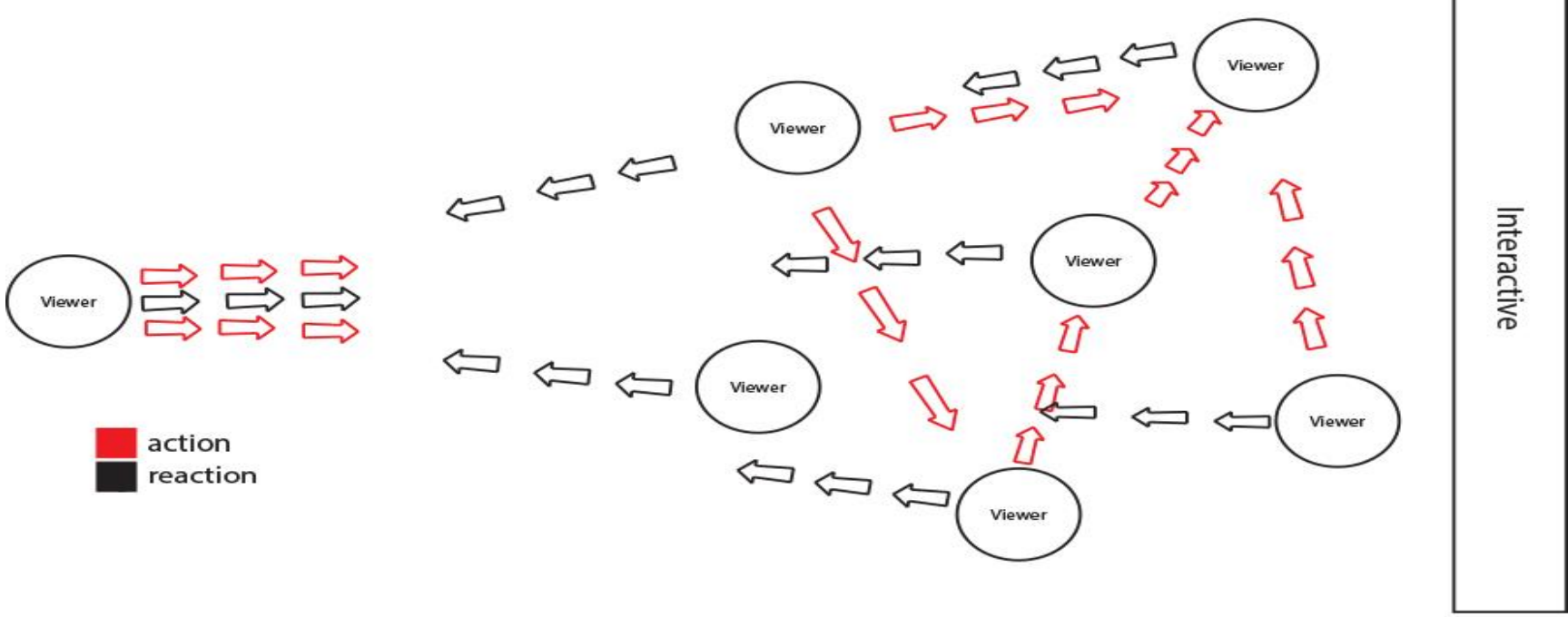

Figure 2: Diagram illustrating the levels of interaction within the demonstrated system (By the author, 2015)

\section{3: DESCRIPTION}

'Augmented Reality (AR) represents the future of the digital integrated museum experience. There is considerable scope for providing engaging and interactive experiences when using AR combined with traditional museum practices' (Patel \& Tuck 2014).

This demonstration is in essence a participatory experience in which the viewer in their interaction with the artwork, will generate a digital sculpture that responds to both individual and collaborative action. Utilising Augmented Reality technology as a conduit to deliver adaptive and self-generating material that responds to the actions of the participants. Contained within the physical space a large-scale artwork will be hung on one wall, this piece will act as a 'natural feature' target, which will instigate an augmented reality response.

Initially a simple grey cube will appear to hover in $3 \mathrm{D}$ in front of the physical artwork, however when a participant interrupts the line of sight between the physical artwork and the device camera this will instigate a reaction and the grey cube will evolve. This evolution will respond directly to the position of the participants, with a huge variety of selfgenerative outcomes that are dependent on their actions and interaction. Collaboration and experimentation is required of the participants in order for the full complexity to be revealed.

\section{4: CONTEXTUALISATION}

'Augmented reality as a medium rather than a technology offers users the possibility to deploy their phones as pocket-sized screens through which surrounding spaces becomes the stage for endless extra layers' (Schavemaker \& Wils 2011).

These extra layers, enriched with digital content, provide the tools for artists to develop narratives and experiences that expand beyond the white cube. This enables the creation of participatory events that question the viewers relationship to both the space and the content contained within.

Augmented Reality in this project enables the integration of generative and participatory content seamlessly within a space, by providing the framework for the viewer to use their physical presence to interface with a self-generative artwork. It seeks to challenge the paradigm of the artist viewer relationship in which the participant is a passive observer.

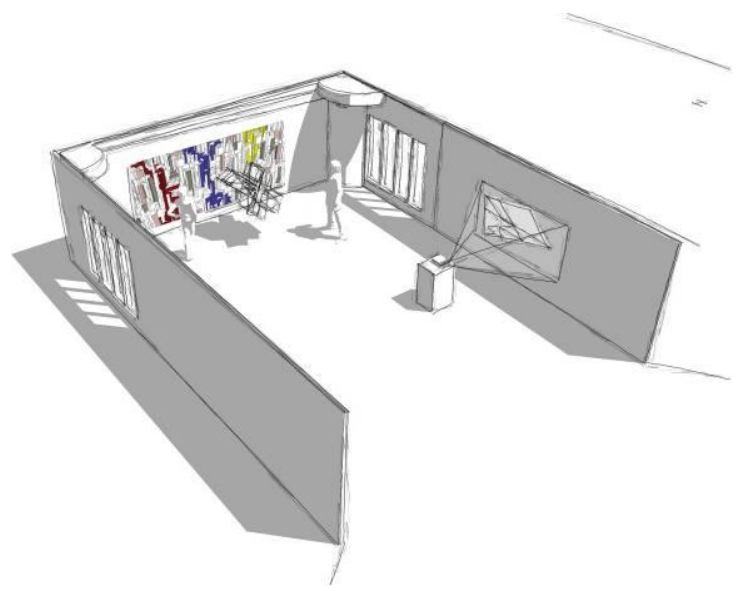

Figure 3: Visualisation (By the author, 2015) 
This project provides an opportunity for the participant to have an immediate and tangible impact upon the output. The interactions and generative reactions can be recorded and disseminated as the participant desires, further disrupting concepts of ownership, authorship and artistic intention and exploring the role of the artist within generative systems

\section{5: DEVELOPMENT}

The key purpose of this piece, as described above, is to provide an interface that both encourages and ultimately necessitates viewers to work collaboratively, to produce a reaction which is responsive to the actions and interactions of multiple participants. During the early stages of development the intention was to provide no instruction or guidance to those who where involved, simply providing a couple of tablets and pointing them at the physical artwork/image target. It was felt, perhaps naively, that the process of discovering the nature of the interaction and through experimentation and teamwork fully revealing the complexity of the artwork that the participatory experience would be enhanced by this journey.

\begin{abstract}
'One of the biggest challenges when designing interactive exhibits occurs where visitors have to follow a particular sequence of actions in order to generate the core phenomenon' (Gammon 2008).
\end{abstract}

Unfortunately and perhaps with hindsight inevitable the majority of those asked to test the piece failed to make the link between their actions and movements and the impact that this had upon the generative response. A few groups did, through both chance and experimentation discover the effects that their presence had, but the vast majority became either frustrated or disinterest before this realisation occurred.

It is obviously a problem when a greater number of users following this participatory experience felt frustrated or disinterested, which was certainly not the meaning that was intended. Following this it seemed appropriate to offer some level of information to guide this process and reduce some of the frustrations that are associated with an inability to control or understand the interface and pull down some of the unexpected barriers that occurred. Printed guidance was produced that detailed the operation of the piece and instructed those viewing of the steps necessary to engage effectively. However this also was not a wholly satisfactory solution, whilst far more participants understood the process and proceeded to interact with the work in the described way much of the collaborative interactions that arose from play and experimentation was lost.

These tests highlighted the shortcoming inherent within the interface, in the first instance with no guidance it became difficult for those taking part to garner a meaningful reaction and when guidance is provided the reactions were, in the most part, simply a reflection of the instructions. With the participant not experimenting and conversing, thus reducing the social interaction that is at the heart of what is trying to be achieved.

It is clear that further development is required. It is the intention to imbed both visual and audio cues within the digital interface to provide feedback, hopefully enabling a more natural and organic journey and leaving scope for experimentation and discovery, but giving sufficient clarity to prevent disaffection.

\section{6: CONCLUSION}

The development of this piece and the testing that has gone on during that development certainly demonstrates that there is potential here to deliver an engaging, multiple participant experience. Albeit one that requires refinement to enable the majority of viewers the opportunity to fully experience this. When observing the test participants it is clear that social interaction and communication is facilitated and that this process is one that, for the most part, augmented the experiences of those involved.

It is the intention that further testing and development of this artwork, both relating to the interface and the overall aesthetic will occur. Through this process a more consistent experience will hopefully emerge that will enable understanding but preserves the feeling of discovery through group endeavour that was so paramount in creating lasting meaning. With the hope, that those taking part in the demonstration will experience this participatory event and that this will deliver one of those powerful epiphanies.

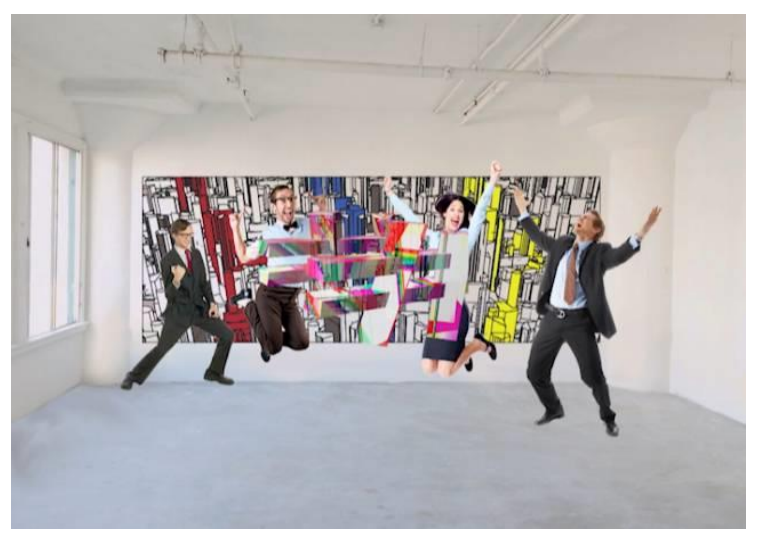

Figure 4: Visualisation By the author, 2015 


\section{REFERENCES}

Falk, J. H. \& Dierking, L. D. (1997) The Museum Experience. Whalesback Books. Washington.

Griggs, S. A. (1990) Perceptions of Traditional and New Style Exhibitions at The Natural History Museum, London. ILVS Review: A Journal of Visitor Behavior, 1(2), 78-90.

Gammon, B. (2008) Seven more lessons learnt in interactive exhibit development. In the Museum Association Conference: Senses working overtime (pp. 22-26). London.

Heath, C. \& Vom Lehn, D. (2010) Interactivity and Collaboration: new forms of participation in museums, galleries and science centres. In Parry, R (Eds), Museums in a Digital Age. Routledge London.

Heath, C., Vom Lehn, D., \& Osborne, J. (2005) Interaction and interactives: collaboration and participation with computer-based exhibits. Public
Understanding of Science, 14, 91-101. Sage, London.

Hein, G. E. (1998) Learning in the Museum. Assessment (vol. 47, p. 203). Routledge, New York.

Huhtamo, E. (2010) On the Origins of the Virtual Museum. In R. Parry (ed.), Museums in a Digital Age. Routledge, London.

Parkin, C. (2014) Chris Milk: The digital artist making music videos fly into the future. The Guardian, p. 30, 18 June.

Patel, R. \& Tuck, D. (2014) Narrative approaches to design multi screen augmented reality experiences. (EVA 2014) Electronic Visualisation and the Arts (pp. 237-243). British Computer Society, London.

Tsoroni, A (2009) 'Technology Fatigue in Digital Interactive Exhibitions', in Engage, vol. 24, pp. 2732. 